\title{
Distinct Contributory Factors Determine Basophil-Allergen Sensitivity in Grass Pollen Rhinitis and in Anaphylactic Wasp Venom Allergy
}

\author{
Peter Korošec Mira Šilar Peter Kopač Renato Eržen Mihaela Zidarn \\ Mitja Košnik \\ University Clinic of Respiratory and Allergic Diseases, Golnik, Slovenia
}

\begin{abstract}
Keywords
IgE $\cdot$ Hymenoptera venom $\cdot$ Grass pollen $\cdot$ House dust mite $\cdot$ Allergen sensitivity Basophils $\cdot$ CD63 Passive lgE sensitisation
\end{abstract}

\begin{abstract}
Background: We sought to determine whether basophil-allergen sensitivity could be transferred to donor basophils by passive IgE sensitisation in allergic rhinitis and anaphylactic Hymenoptera venom hypersensitivity. Methods: We studied 15 wasp venom-, 19 grass pollen- and 2 house dust mite-allergic patients, 2 healthy donors, and 8 wasp venom-allergic donors. In all subjects, we first evaluated the initial basophil response to wasp venom, grass pollen, or house dust mite allergen. Donor basophils were then stripped, sensitised with the different patients' serum IgE, and challenged with the corresponding allergen. The CD63 response of donor basophils was then compared with initial basophil responses. Results: In wasp venom-allergic subjects, the IgE transfer did not reflect the initial basophil-allergen sensitivity, because the venom IgE of subjects with high or low basophil sensitivity induced comparable responsiveness in healthy donor basophils. Furthermore, vice versa, when we sensitised the donor basophils of wasp venom-allergic individuals with different wasp venom or house dust mite lgE, we demonstrated
\end{abstract}

that their response was predictable by their initial basophil allergen sensitivity. In the rhinitis allergy model, the $\operatorname{lgE}$ transfer correlated with the patients' initial basophil responsiveness because the grass pollen IgE of the subjects with high basophil allergen sensitivity induced significantly higher responsiveness of donor basophils than the lgE of subjects with initially low basophil allergen sensitivity. Conclusions: Our results suggest that basophil allergen sensitivity evaluated by flow-cytometric CD63 analysis depends on two distinct contribution factors. In anaphylactic Hymenoptera allergy, the major factor was intrinsic cellular sensitivity, whereas in pollen allergy, the major factor was allergen-specific $\lg E$ on the cell surface.

(c) 2016 S. Karger AG, Basel

\section{Introduction}

The IgE-mediated activation of basophils and mast cells following exposure to an allergen and the release of mediators is a critical point in the allergic response. At the cellular level, this response corresponds to 2 independent variables [1]. Basophil reactivity is the maximal response to allergen stimulation at the plateau of the dose-response curve. The basophil threshold of allergen sensitivity represents the lowest or half-maximum part of the dose-re-

\section{KARGER}

(c) 2016 S. Karger AG, Basel

E-Mail karger@karger.com

www.karger.com/iaa
Correspondence to: Prof. Peter Korošec

Laboratory for Clinical Immunology and Molecular Genetics

University Clinic of Respiratory and Allergic Diseases

Golnik 36, SI-4204 Golnik (Slovenia)

E-Mail peter.korosec@klinika-golnik.si 
sponse curve $[2,3]$ and could also be analysed by CD-sens calculation [2]. The major determinants of allergic basophil responses are IgE antibodies, which are critical to the induction of the response, the expression of a high-affinity receptor for IgE (FceRI), which is tightly regulated by IgE antibodies, and very likely intracellular factors, such as factors produced from a signal transduction cascade in which spleen tyrosine kinase (Syk) plays a central role [4, 5]. The cellular effect of IgE antibodies was recently explored in vivo using omalizumab to manipulate the $\operatorname{IgE}$ levels. In cat allergy and grass pollen allergy, the basophil response is markedly decreased via the suppression of allergen-specific IgE antibodies; this decrease is significantly correlated with a decrease in clinical signs and symptoms during an experimental allergen challenge $[6,7]$. Unexpectedly, in anaphylactic peanut allergy after omalizumab treatment, the decreased basophil and clinical response was not evident in the majority of patients $[8,9]$.

In patients who develop anaphylaxis from a Hymenoptera sting, many studies have reported no clear relationship between the levels of allergen-specific IgE and the presence, absence, or severity of the clinical response to the allergen, which was also evident when the sensitisation was confirmed using a major venom recombinant allergens [10-13]. Previous reports have described patients with a history of systemic reactions but negative serum venom-specific IgE and skin test results in response to a Hymenoptera sting [11, 14]. Moreover, these patients can subsequently experience another severe reaction to a sting [11]. In fact, in cases of fatal sting anaphylaxis, venom-specific IgE is very low or even undetectable in more than $30 \%$ of patients [15].

In patients with pollen allergy, allergen-specific IgE levels were more closely associated with the severity of allergic seasonal rhinitis or with an increased likelihood of atopic wheezing, especially in the case of a high specific/ total IgE ratio or positive IgE reactivity to major pollen species-specific recombinant allergens, such as Phl p 1 and $\mathrm{p} 5$ or Bet $\mathrm{v} 1$ [16-19]. The recombinant allergens help to avoid IgE-binding to profilins and/or cross-reactive carbohydrates with no or limited clinical significance [20].

To further explore the relationship between IgE antibodies and the basophil sensitivity in different allergic diseases, we set up a controlled experimental design of passive IgE sensitisation of stripped donor basophils that was first used in the 1980s for histamine release assays [21] and then adapted for a basophil activation assay [22]. First, we selected subjects that were allergic to wasp venom or grass pollen with significantly different basophil allergen sensitivity. We then transferred their serum $\operatorname{IgE}$ antibodies to donor basophils of healthy or wasp venomallergic subjects by passive IgE sensitisation and evaluated whether basophil responses of sensitised donor basophils were comparable with the patients' initial basophil responsiveness. We were interested in whether specific $\operatorname{IgE}$ antibodies in these diseases are a major predictor of basophil allergen sensitivity or whether this response is also significantly related to the intrinsic sensitivity of basophils. To confirm the results related to the role of the intrinsic sensitivity of basophils, we selected another group of wasp venom-allergic basophil donors, who were sensitised with the sera of house dust mite-allergic subjects.

\section{Patients and Methods}

\section{Study Groups}

The first study group included 15 wasp venom-allergic subjects ( 8 women and 7 men with a mean age of 37 years and an age range of 18-64 years) with a history of systemic sting reactions of Mueller grades I-IV ( 3 grade I, 3 grade II, 5 grade III, and 4 grade IV). All of the subjects had positive, specific IgE to wasp venom (median $13.1 \mathrm{kU} / \mathrm{L}$; range: $3.1-24.9$ ), and 13 of them (87\%) had also a positive, specific response to the major recombinant allergen Ves v 5 (median $4.3 \mathrm{kU} / \mathrm{L}$; range: $0.6-32.9$ ). The second study group included 19 grass pollen-allergic subjects (6 women and 13 men with a mean age of 37 years and an age range of 18-64 years) with a history of allergic rhinitis without asthma. All of the subjects had positive specific IgE to timothy grass (median $21.5 \mathrm{kU} / \mathrm{L}$; range: $1.3-$ 91.6) and to the major recombinant grass allergens $\mathrm{rPhl} p 1$ and $\mathrm{p}$ $5 \mathrm{~b}$ (median $29 \mathrm{kU} / \mathrm{L}$; range: $0.4-72.4$ ). We tested individual sera of those 2 study groups with basophils of healthy non-allergic donors ( 1 man and 1 woman aged 41 and 27 years, respectively), and wasp venom-allergic donors ( 2 men, 27 and 51 years old, respectively; 1 with grade I and 1 with grade II reactions; specific IgE to wasp venom 0.57 and $9.7 \mathrm{kU} / \mathrm{L})$. A third study group consisted of 6 wasp venom-allergic donors ( 2 women and 4 men with a mean age of 51 years and an age range of 24-68 years; 1 grade II, 2 grade III, and 3 grade IV reactions; specific IgE to wasp venom: median $11.9 \mathrm{kU} / \mathrm{L}$; range: $1.2-21.9 ; 5$ of them had positive specific IgE to Vesp v 5: median $1.8 \mathrm{kU} / \mathrm{L}$; range: $0.35-69.2$ ), and the basophils of those donors were sensitised with sera of 2 house dust mite-allergic subjects ( 1 women and 1 man, 20 and 28 years old, respectively; specific IgE to Dermatophagoides pteronyssinus: 12 and $19.1 \mathrm{kU} / \mathrm{L}$ ). The data of 3 wasp venom-allergic donors have been published recently [23]. At serum inclusion or donor testing, none of the subjects had been treated with immunotherapy. All of the subjects provided written informed consent for participation in the study. The study was approved by the Slovenian National Medical Ethics Committee.

\section{IgE Measurements}

The levels of IgE antibodies to wasp venom (i3), major wasp venom allergen rVes v 5 (i209), timothy grass (g6), major grass pollen allergens rPhl p 1 and p 5b (g213), D. pteronyssinus (d1), and total IgE were quantified using the ImmunoCAP system (Thermo Fisher Scientific, Waltham, MA, USA).
Korošec/Šilar/Kopač/Eržen/Zidarn/ Košnik 


\section{Passive IgE Sensitisation}

Peripheral blood mononuclear cells (PBMCs) from the heparinised blood of the healthy or wasp venom-allergic donors were first separated by a Ficoll-Paque (GE Healthcare Bio-Sciences AB, Uppsala, Sweden) density gradient centrifugation according to the manufacturer's instructions and washed twice with RPMI 1640 (Gibco, Grand Island, NY, USA). From here on to the sensitisation step, everything was kept in an ice bath or at $4^{\circ} \mathrm{C}$. PBMCs were first washed twice with RPMI 1640 with $0.5 \%$ BSA (Sigma-Aldrich, St. Louis, MO, USA) (RPMI-BSA) and then treated with $\mathrm{pH} 3.9$ lactic acid buffer [21] containing $10 \mathrm{~mm}$ lactic acid, $130 \mathrm{mM} \mathrm{NaCl}$, and $5 \mathrm{~mm} \mathrm{KCl}$ (Buhlmann Laboratories, Basel, Switzerland) for 3.5 $\mathrm{min}$. The stripping process was stopped by adding RPMI-BSA and PBS-TrisBase (ImmunoConcepts, Sacramento, CA, USA) and washed twice with RPMI-BSA.

The cells were resuspended and sensitised in a solution containing $40 \%$ sera from subjects that were allergic to wasp venom, grass pollen, or house dust mites, RPMI-BSA, 8 mM EDTA (Sigma-Aldrich), and $5 \mathrm{IU} / \mathrm{mL}$ heparin (Krka, Novo mesto, Slovenia) in a heated water bath for $90 \mathrm{~min}$ at $37^{\circ} \mathrm{C}$. The incubation was stopped by adding cold RPMI-BSA, and cells were washed twice with RPMI-BSA at $4^{\circ} \mathrm{C}$ and resuspended in RPMI-BSA before being immediately used in a basophil activation assay [22]. For the wasp venom-allergic donor basophils, the response to wasp venom was also tested after IgE removal before passive sensitisation, in which no basophil activation was demonstrated.

\section{Basophil Activation Assay}

The basophil activation test was performed as previously described $[3,14,19,23]$. Briefly, whole blood (with heparin anticoagulant) or sensitised PBMCs was preincubated in basophil stimulation buffer with IL-3 (Buhlmann Laboratories) containing a final concentration of 0.1 and 1 or $0.01-1 \mu \mathrm{g} / \mathrm{mL}$ of wasp venom [1-3] (Hal Allergie) or $0.01-10$ or $100 \mu \mathrm{g} / \mathrm{mL}$ of grass pollen allergen (Aquagen ${ }^{\circledR} \mathrm{SQ}$, freeze-dried allergen extract of grass pollen mix L299; ALK Abello, Madrid, Spain) or 1.665-333.3 ng/mL of $D$. pteronyssinus extract (Buhlmann Laboratories) at $37^{\circ} \mathrm{C}$ for $15 \mathrm{~min}$. We also included anti-FceRI mAb $(0.55 \mu \mathrm{g} / \mathrm{mL}$; Buhlmann Laboratories) and fMLP controls ( $2 \mu \mathrm{M}$; Sigma-Aldrich). Degranulation was stopped by chilling on ice, after which FITCconjugated anti-CD63 mAb, PE-conjugated anti-CD123 mAb, and PerCP-conjugated anti-HLA-DR mAb (BD Biosciences, San Jose, CA, USA) were added and incubated for $20 \mathrm{~min}$ on ice. Finally, whole-blood probes were lysed, washed, fixed, and analysed within $2 \mathrm{~h}$ on a FACSCalibur flow cytometer (BD Biosciences). The data were acquired using a FL2 threshold that eliminated most of the CD123-negative cells, and at least 400 CD123-positive cells were acquired per sample. The basophils were identified as low-side scatter, CD123-positive, and HLADR-negative cells, and the percentage of degranulated basophils was quantified in FL1 (CD63). In some experiments, the basophil sensitivity was also evaluated by $\mathrm{CD}$-sens calculation as previously described $[2,6,19,23]$. Briefly, basophil CD63 dose-response curves for single patients were plotted using Magellan 7.1 data analysis software (Tecan, Männedorf, Switzerland), followed by the CD-sens calculation, which is the inverse value of the allergen concentration (in $\mu \mathrm{g} / \mathrm{mL}$ ) at $50 \%$ of individual maximal response multiplied by 100 .

Distinct Factors Determine

Basophil-Allergen Sensitivity

\section{IgE and FceRI Cell Surface Expression}

The cell surface expression of IgE and FceRI receptors was evaluated as previously described [2,23-25]. Briefly, the number of IgE molecules and FceRI receptors per basophil were calculated using an FITC-conjugated antibody to IgE (Miltenyi Biotec, Teterow, Germany) or FceRI (eBioscience, San Diego, CA, USA), and compared to a standard curve of calibration beads (QIFIKIT; DakoCytomation, Glostrup, Denmark). With assigned molecules of equivalent FITC fluorochrome values for the fluorescent bead populations, arbitrary units of mean FITC fluorescence intensity could be transformed into absolute units.

\section{Statistical Analyses}

The distribution of the data was assessed using the D'Agostino and Pearson omnibus normality test. Based on the distribution of the data, we used a $t$ test or a Mann-Whitney test, and the results were presented as means or medians and ranges. The correlation coefficients were calculated using the Spearman rank correlation coefficient. In the case of binominal outcomes, we used the $\chi^{2}$ test without the Yates correction. Probability values of $p<0.05$ were considered significant. If not stated otherwise, the results were presented as medians and ranges. The analyses were performed using GraphPad Prism 5.

\section{Results}

\section{Selection and Comparison of Healthy and Wasp Venom-Allergic Donors}

We first compared the response of the basophils of 2 healthy donors after sensitisation with the sera of 2 selected subjects that were allergic to wasp venom or grass pollen. The basophils of the 1 st donor had $84 \times 10^{3} \mathrm{IgE}$ and $136 \times 10^{3}$ FceRI molecules per cell. The basophils of the second donor had $79 \times 10^{3} \mathrm{IgE}$ and $73 \times 10^{3} \mathrm{Fc \varepsilon RI}$ molecules per cell. Both of the donors had a negative initial basophil response to wasp and grass pollen allergens and a positive response to both fMLP and anti-FceRI control stimuli (Fig. 1a, b). After passive IgE sensitisation, both of the donors showed highly comparable responsiveness to wasp or grass pollen allergen stimuli and to both fMLP and anti-FceRI control stimuli (Fig. 1c, d). Therefore, only 1 healthy donor was selected for further experiments (healthy donor 1 ).

We selected 2 wasp venom-allergic basophil donors, a donor with an initially high basophil allergen threshold sensitivity (CD63 response to $0.1 \mu \mathrm{g} / \mathrm{mL}$ of venom $99 \%$ and to $1 \mu \mathrm{g} / \mathrm{mL} 98 \%$ ) and a donor with an initially low basophil allergen threshold sensitivity $(6 \%$ to $0.1 \mu \mathrm{g} / \mathrm{mL}$ and $70 \%$ to $1 \mu \mathrm{g} / \mathrm{mL}$ ). The high-sensitivity wasp venomallergic donor basophils had $138 \times 10^{3} \mathrm{IgE}$ and $111 \times 10^{3}$ FceRI molecules per cell. The low-sensitivity wasp venom-allergic donor basophils had $125 \times 10^{3} \mathrm{IgE}$ and $114 \times$ $10^{3}$ FceRI molecules per cell. 


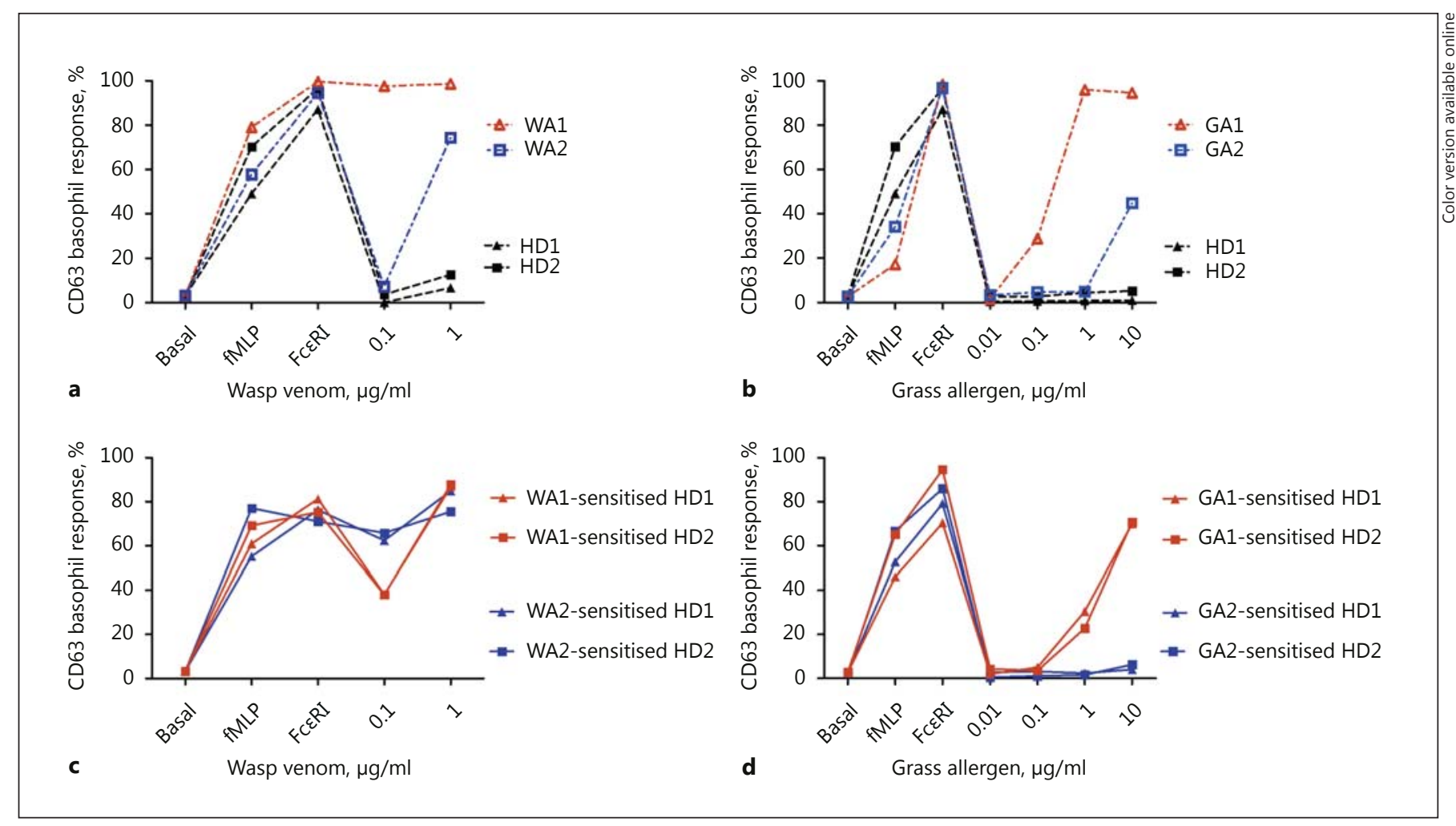

Fig. 1. a-d Initial CD63 response of basophils in 2 healthy donors (HD) and 2 wasp-allergic (WA; a) or grassallergic subjects $(\mathrm{GA} ; \mathbf{b})$ and the response of HD basophils after passive IgE sensitisation with the sera of 2 WA (c) or GA subjects (d). Basal, stimulation buffer with IL-3 alone; fMLP, stimulation with N-formyl-Met-Leu-Phe; FceRI, stimulation with $0.55 \mu \mathrm{g} / \mathrm{mL}$ anti-FceRI mAb.

We then selected another 6 wasp venom-allergic basophil donors. We first evaluated whole dose-response curves of initial wasp venom basophil responses in these donors. The curves and thus threshold basophil allergen sensitivity differed between donors. Five donors demonstrated comparable IgE levels (mean [range] = 134 [97$166] \times 10^{3}$ ) and FceRI (mean [range] $=122[87-141] \times$ $10^{3}$ ) molecules per basophil. One donor had a lower IgE level $\left(23 \times 10^{3}\right)$ and a lower number of FceRI $\left(26 \times 10^{3}\right)$ molecules per cell.

Passive Sensitisation of Healthy Donor Basophils with Wasp Venom IgE

Of the 15 wasp venom-allergic subjects whose sera were used for passive IgE sensitisation, 7 subjects showed initially high basophil allergen sensitivity and 9 showed low basophil allergen sensitivity. Sensitivity was determined according to the CD63 response to the submaxi$\mathrm{mal}(0.1 \mu \mathrm{g} / \mathrm{mL})$ venom concentration, as previously described $[3,14,26,27]$. In the high-sensitivity subgroup, the median basophil response to $0.1 \mu \mathrm{g} / \mathrm{mL}$ was $72 \%$ (range 58-99), whereas in the low-sensitivity subgroup, the response was $11 \%$ (range $0.7-30)(p=0.0003$; Fig. 2). The basophil response to the high venom concentration $(1 \mu \mathrm{g} / \mathrm{mL})$ was comparable in both subgroups: (median [range] $=90 \%$ [59-99] vs. 84\% [69-96]). The CD63 response to fMLP (median [range]: $49 \%$ [17-79] vs. $50 \%$ [14-83]) and anti-FceRI mAb (median [range]: 86\% [48$100]$ vs. 94\% [73-99]) was also comparable.

After passive IgE sensitisation of healthy donor basophils with the sera of the wasp venom-allergic subjects with an initially high or low basophil allergen sensitivity, the median CD63 response to $0.1 \mu \mathrm{g} / \mathrm{mL}$ venom was $37 \%$ (range: 15-80) for sera from the high-sensitivity subgroup and 34\% (range: 20-91) for sera from the low-sensitivity subgroup (Fig. 2). This highly comparable CD63 response of healthy donor basophils was also evident at $1 \mu \mathrm{g} / \mathrm{mL}$ venom, with a median response of $61 \%$ (range: 28-93) and of 64\% (range: 20-91), respectively.
Korošec/Šilar/Kopač/Eržen/Zidarn/ Košnik 


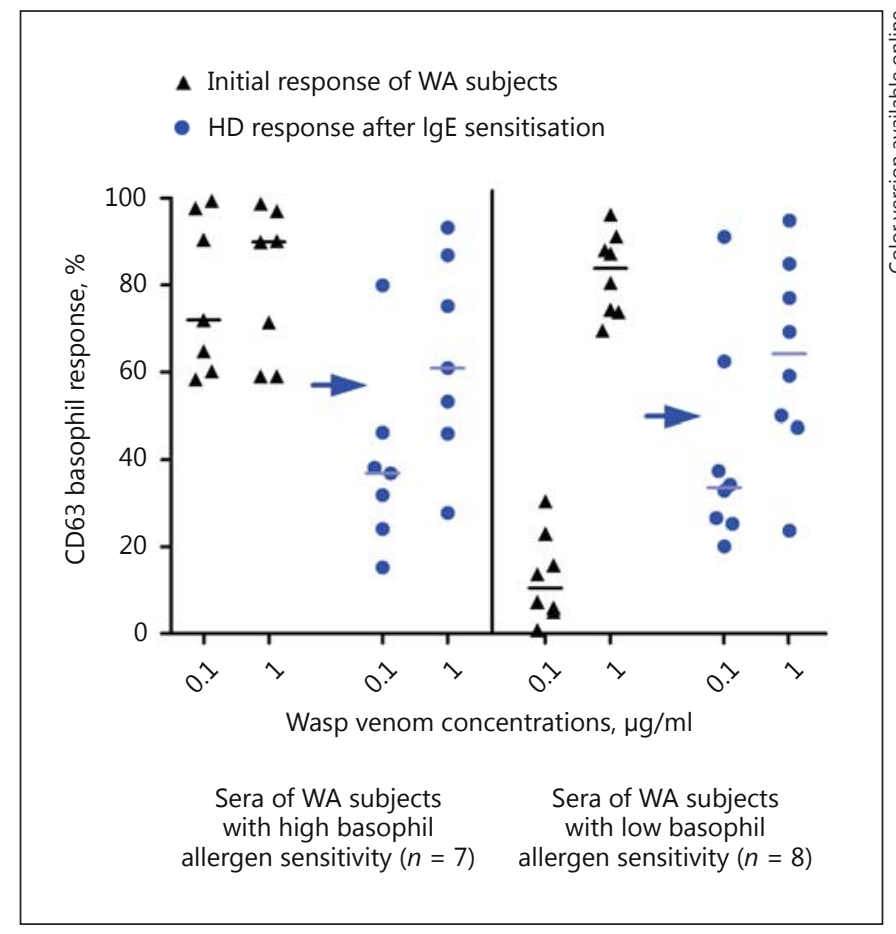

Fig. 2. Wasp venom CD63 response after passive IgE sensitisation of healthy donor (HD) basophils (arrow) with the sera of 7 waspallergic (WA) subjects showed initially high basophil allergen sensitivity, and the sera of 9 WA subjects showed initially low basophil allergen sensitivity. The horizontal line indicates the median.

Passive Sensitisation of Healthy Donor Basophils with Grass Pollen IgE

Of the 19 grass pollen-allergic subjects whose sera were used for passive IgE sensitisation, 11 subjects showed initially high allergen threshold sensitivity, and 8 showed low basophil-allergen threshold sensitivity. The basophil sensitivity was evaluated according to the CD63 response to four different grass pollen log allergen concentrations, as previously described [19]. The most prominent differences were at the allergen concentrations of 0.1 and $1 \mu \mathrm{g} /$ $\mathrm{mL}$ because in the high-sensitivity subgroup, the median CD63 responses to 0.1 and $1 \mu \mathrm{g} / \mathrm{mL}$ allergen were 8 and 89\% (range: 6-29 and 58-99), and in the low-sensitivity subgroup, responses were $3 \%$ (range $1-8$ ) and $8 \%$ (range: $3-29$ ), respectively ( $p \leq 0.001$; Fig. 3a, b). These differences were also demonstrated by CD-sens analysis (median [range]: 330 [142-955] vs. 37 [17-66]; $p=0.0003$; Fig. 3c). The median responses to 0.01 and $10 \mu \mathrm{g} / \mathrm{mL}$ grass allergen were 4 and $98 \%$ (range: $1-29$ and $88-100$ ) for the high-sensitivity subgroup and 3 and $85 \%$ (range: $1-3$ and 33-95) for the low-sensitivity subgroup, respectively

Distinct Factors Determine

Basophil-Allergen Sensitivity
(Fig. 3a, b). The response to control stimuli was comparable (fMLP: median 53 vs. $34 \%$ [range: $3-86$ vs. $10-86$ ]; anti-FceRI mAb: median 98 vs. 97\% [range: 82-99 vs. $23-$ 100]) between both subgroups.

After passive IgE sensitisation, the sera of grass pollen-allergic subjects with initially high basophil-allergen threshold sensitivity induced a significantly higher healthy donor basophil CD63 response as did IgE sensitisation with the sera of subjects with initially low basophil allergen sensitivity (Fig. 3a, b). Thus, the median CD63 response to $1 \mu \mathrm{g} / \mathrm{mL}$ allergen was $46 \%$ (range: 5-70) for sera from the high-sensitivity subgroup and $8 \%$ (range: 4-34) for sera from the low-sensitivity subgroup $(p=0.004)$. Similar significant differences were also found for the response to $10 \mu \mathrm{g} / \mathrm{mL}$ (median [range]: 69\% [22$85]$ vs. $23 \%$ [14-85]; $p=0.002$ ) and to $100 \mu \mathrm{g} / \mathrm{mL}$ (median [range]: $75 \%$ [33-89] vs. $38 \%$ [25-90]) $(p=0.02$; Fig. 3a, b) grass allergen. Furthermore, this differences were also shown by $\mathrm{CD}$-sens analysis (median [range]: 122 [7-538] vs. 18 [2-67]; $p=0.007$; Fig. 3c). The median responses to grass allergen concentrations of 0.01 and $0.1 \mu \mathrm{g} / \mathrm{mL}$ were 4 and $10 \%$ (range: $1-8$ and $3-32$ ) for the high-sensitivity subgroup and 4 and $4 \%$ (range: $0.4-7$ and 1-20) for the low-sensitivity subgroup.

\section{Passive Sensitisation of Wasp Venom-Allergic Donor \\ Basophils with Wasp Venom IgE}

For passive sensitisation, we included the sera of 4 wasp venom-allergic subjects that showed initially a high basophil allergen threshold sensitivity (median 85\% [range 60-99] at $0.1 \mu \mathrm{g} / \mathrm{mL}$ ) and the sera of 3 subjects that showed initially a low basophil allergen threshold sensitivity (median 5\% [range $1-23$ ] at $0.1 \mu \mathrm{g} / \mathrm{mL} ; p=0.002$ ). In both subgroups, the CD63 response to $1 \mu \mathrm{g} / \mathrm{mL}$ venom (median [range]: 84\% [59-100] vs. 74\% [69-88]) was comparable.

After passive IgE sensitisation of high-sensitivity wasp-allergic donor basophils, the median CD63 response to $0.1 \mu \mathrm{g} / \mathrm{mL}$ venom was high at $79 \%$ (range: $74-$ 82 ) for sera from the high-sensitivity subgroup and $74 \%$ (range: 72-87) for sera from the low-sensitivity subgroup (Fig. 4a). The median response to $1 \mu \mathrm{g} / \mathrm{mL}$ venom was 77\% (range: 69-100) and 79\% (range: 79-81), respectively. By contrast, after the passive IgE sensitisation of the low-sensitivity wasp-allergic donor basophils, the median CD63 response to $0.1 \mu \mathrm{g} / \mathrm{mL}$ venom was low at $28 \%$ (range: 5-46) for the sera from the high-sensitivity subgroup and 15\% (range: $12-25)$ for the sera from the lowsensitivity subgroup ( $p \leq 0.002$; Fig. $4 b$ ). The low-sensitivity donor response to $1 \mu \mathrm{g} / \mathrm{mL}$ venom was also lower, 


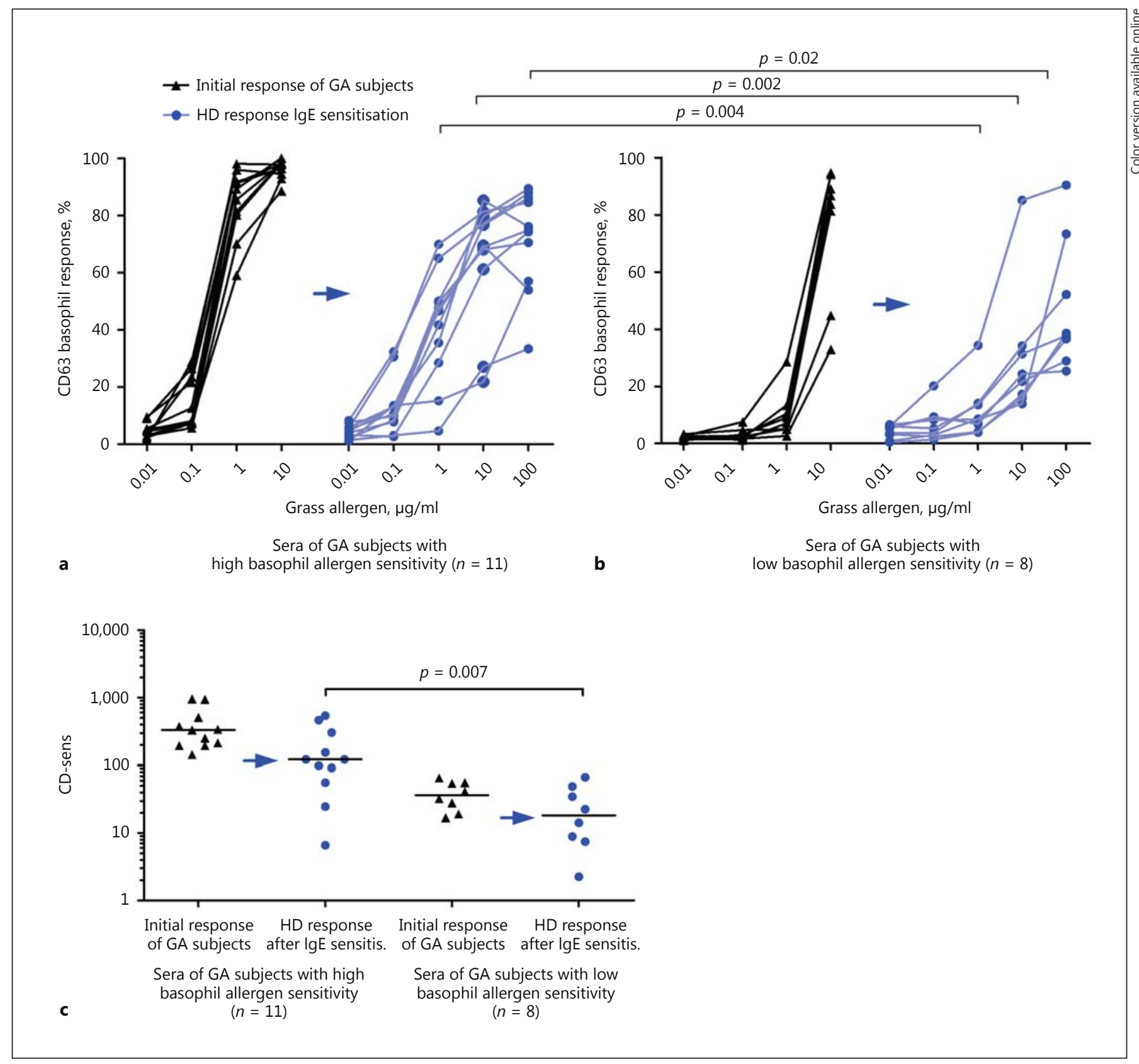

Fig. 3. a-c Grass pollen CD63 response after passive IgE sensitisation (arrow) of healthy donor (HD) basophils with the sera of 11 grass-allergic (GA) subjects showed initially high basophil allergen sensitivity (a), and sera of 8 GA subjects showed initially low basophil allergen sensitivity (b). $\mathbf{c}$ For both subgroups, we also performed CDsens analysis. The horizontal line indicates the median. CD-sens, the inverse value of the allergen concentration (in $\mu \mathrm{g} / \mathrm{mL}$ ) at $50 \%$ of individual maximal response multiplied by 100 .

with a median of 55\% (range: $40-78$ ) and 39\% (range: $34-59$ ), respectively ( $p=0.01$; Fig. $4 \mathrm{~b}$ ). Comparably low results were also found after passive IgE sensitisation of healthy donor basophils with a median response of $34 \%$ (range: $15-46)$ and $5 \%$ (range: $1-22$ ) at $0.1 \mu \mathrm{g} / \mathrm{mL}$ venom and $57 \%$ (range: $46-75$ ) and 59\% (range: $47-61$ ) at $1 \mu \mathrm{g} /$ $\mathrm{mL}$ venom, respectively (Fig. $4 \mathrm{c}$ ). There was a trend to a higher CD63 response for sera from the high- (55 and 


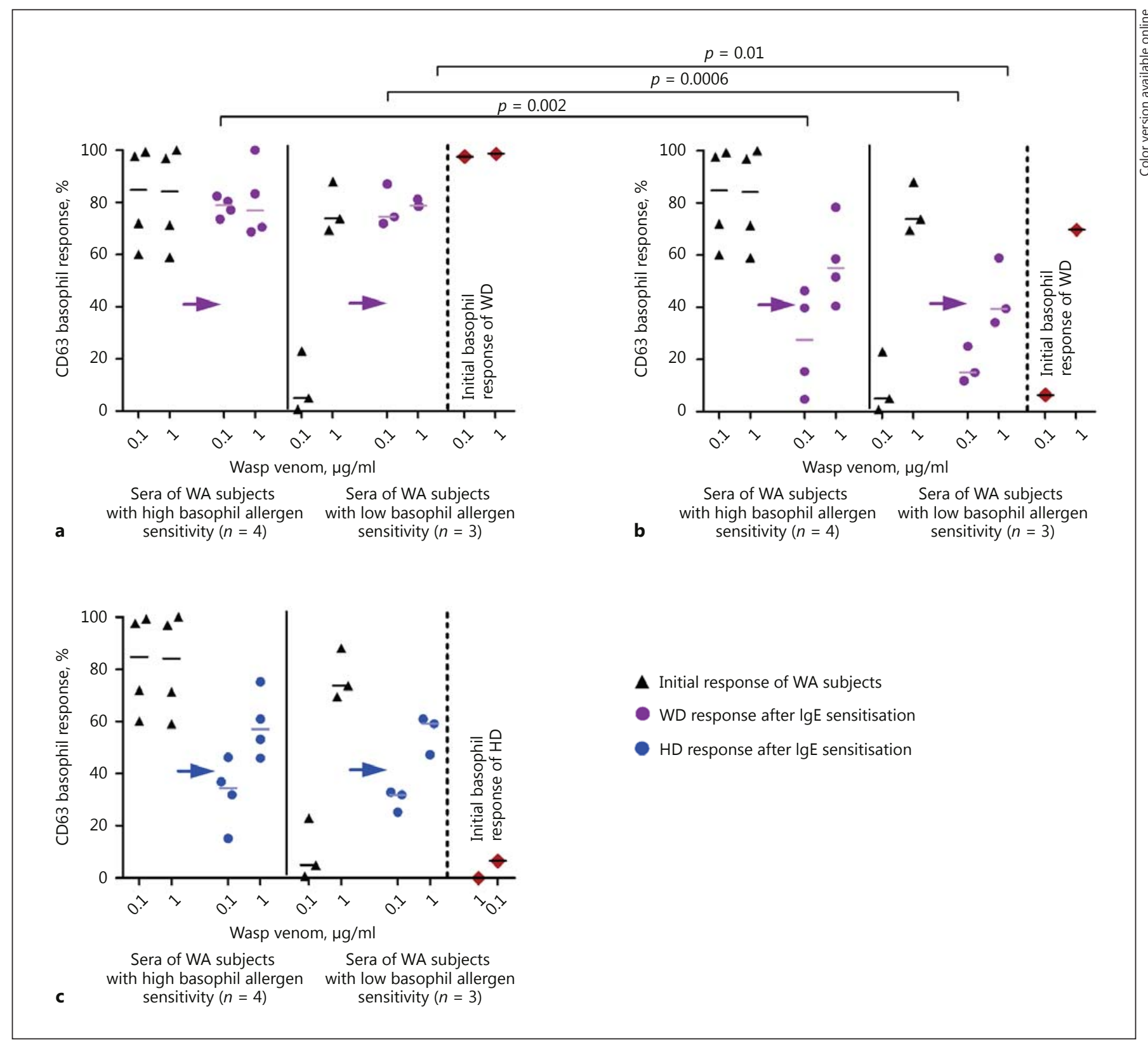

Fig. 4. a-c Wasp venom CD63 response after passive IgE sensitisation of high-sensitivity (a) and of low-sensitivity wasp-allergic (WA) basophils (b) and of healthy donor (HD) basophils (c) with the sera of 4 WA subjects showed initially high basophil allergen sensitivity, and sera of 3 WA subjects showed initially low basophil allergen sensitivity. WD, wasp-allergic donor. The horizontal line indicates the median. Arrows indicate IgE sensitisation of high-sensitivity (a) and low-sensitivity WA basophils (b) and of HD basophils (c).

$28 \%$ at 1 and $0.1 \mu \mathrm{g} / \mathrm{mL}$, respectively) versus low-sensitivity (39 and $15 \%$ at 1 and $0.1 \mu \mathrm{g} / \mathrm{mL}$, respectively) subgroups after passive IgE sensitisation of low-sensitivity wasp-allergic donor basophils (Fig. 4b), but the differences were not significant.

Distinct Factors Determine Basophil-Allergen Sensitivity
Passive Sensitisation of Wasp Venom-Allergic Donor Basophils with House Dust Mite IgE

For passive IgE sensitisation, we included the sera of 2 house dust mite-allergic subjects who showed similar initial basophil responses (from 1.665 to $333.3 \mathrm{ng} / \mathrm{mL}$ of 


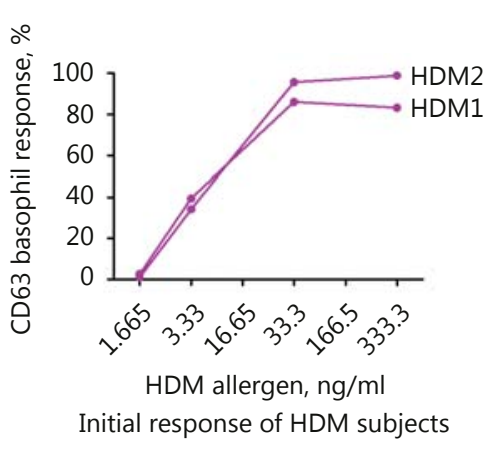

Initial response of HDM subjects
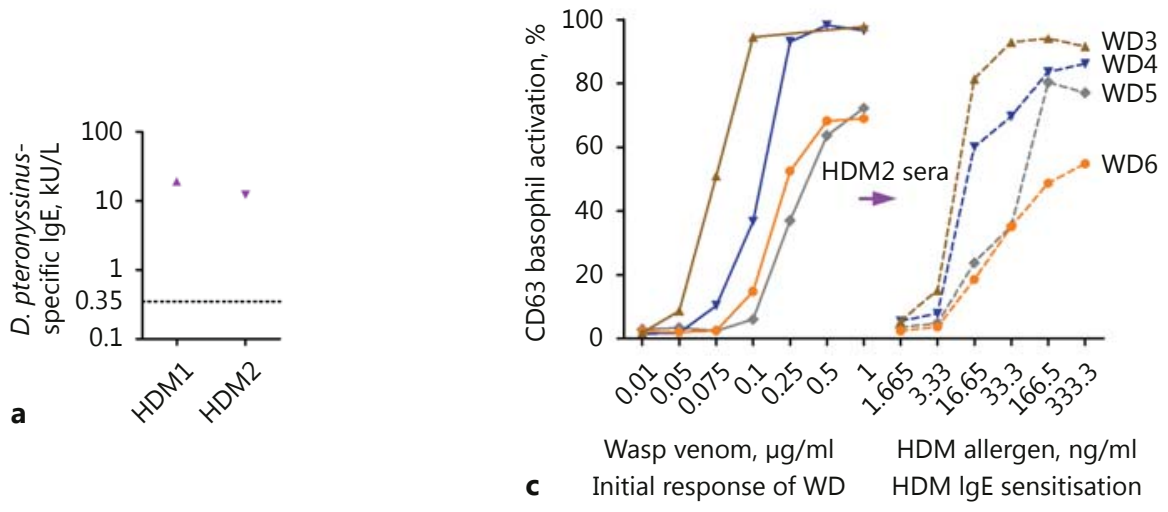

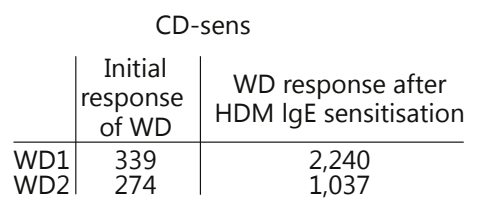
Wasp venom, $\mu \mathrm{g} / \mathrm{ml}$
Initial response of WD
HDM lgE sensitisation

\begin{tabular}{c|c|c}
\multicolumn{3}{c}{ CD-sens } \\
& $\begin{array}{c}\text { Initial } \\
\text { response } \\
\text { of WD }\end{array}$ & $\begin{array}{c}\text { WD response after } \\
\text { HDM lgE sensitisation }\end{array}$ \\
\hline WD3 & 1,351 & 14,206 \\
WD4 & 909 & 9,128 \\
WD5 & 403 & 3,026 \\
WD6 & 595 & 4,090
\end{tabular}

Fig. 5. a House dust mite (HDM) CD63 response and specific IgE level in $2 \mathrm{HDM}$-allergic subjects. b HDM CD63 response and CDsens after passive IgE sensitisation of basophils of 2 wasp-allergic donors (WD1 and WD2) with the sera of the 1st HDM subject (HDM1) and comparison to their initial wasp venom CD63 response and CD-sens. c HDM CD63 response and CD-sens after

house dust mite allergen was 1.1 and 2.6, 34 and 39, 86 and 96 , and 83 and $99 \%$, respectively) (Fig. 5a). With the 1 st sera, we sensitised the basophils of 2 (Fig. 5b) and with the 2 nd sera the basophils of 4 wasp venom-allergic donors (Fig. 5c). In these experiments, we showed that the response curves to house dust mite stimulation after passive sensitisation of donor basophils corresponded to the donor initial basophil response to wasp venom (Fig. 5b, c). This was also clearly demonstrated by CD-sens analysis, which indicated that the donors with higher CD-sens and thus higher basophil sensitivity also showed higher CD-sens and basophil sensitivity after passive sensitisation (Fig. 5b, c). Furthermore, we showed a highly significant positive correlation ( $\mathrm{R}$ coefficient of $1, \mathrm{p}=0.003$ ) passive IgE sensitisation of basophils of 4 WD subjects (WD3WD6) with the sera of the 2nd HDM subject (HDM2) and comparison to their initial wasp venom CD63 response and CD-sens. CD-sens, the inverse value of the allergen concentration (in $\mu \mathrm{g} /$ $\mathrm{mL}$ ) at $50 \%$ of individual maximal response multiplied by 100 . Arrow indicates HDM1 (b) and HDM2 (c) IgE sensitisation. between CD-sens after passive sensitisation and initial CD-sens (Fig. 6).

\section{Serum-Specific IgE, Specific IgE/Total IgE Percentage Ratio and IgE and FceRI Cell Surface Expression Analysis}

In wasp venom-allergic subjects whose sera were used for passive IgE sensitisation, the serum-specific IgE to wasp venom and $\mathrm{rVes} v 5$ and the total IgE did not show any significant differences between the high- and lowsensitivity subgroups: (median [range]: $13.1 \mathrm{kU} / \mathrm{L}$ [3.9$24.9]$ vs. $12.9 \mathrm{kU} / \mathrm{L}[3.1-31] ; 2.9 \mathrm{kU} / \mathrm{L}[<0.35-22.9]$ vs. 8.2 kU/L [<0.35-32.9]; 84 kU/L [18-404] vs. 202 kU/L [261,332], respectively) (Fig. 7a). The percentage of IgE an-
96

Int Arch Allergy Immunol 2016;171:89-101 DOI: $10.1159 / 000452102$
Korošec/Šilar/Kopač/Eržen/Zidarn/ Košnik 


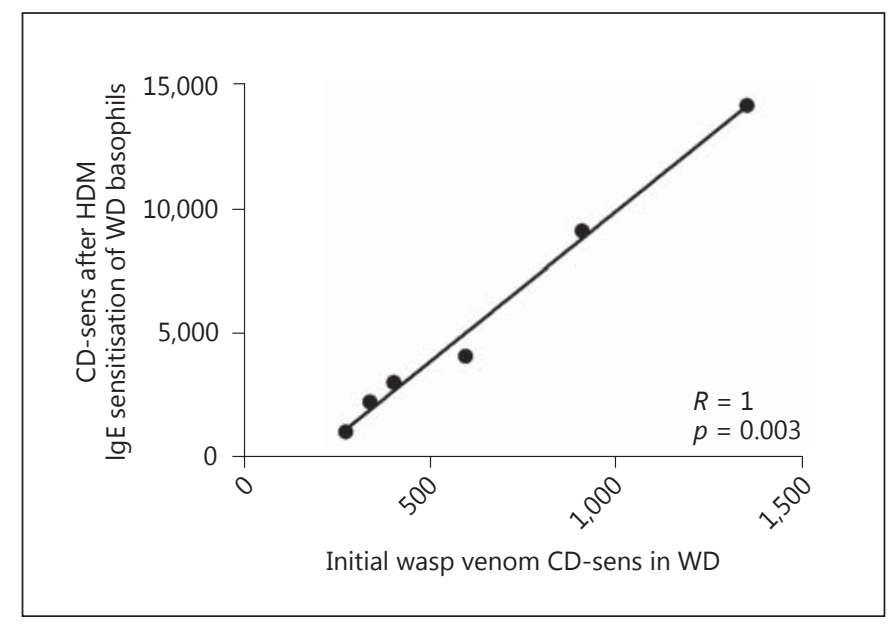

Fig. 6. Correlation between CD-sens after passive house dust mite (HDM) IgE sensitisation and initial wasp venom CD-sens in 6 wasp-allergic donors (WD). CD-sens, the inverse value of the allergen concentration (in $\mu \mathrm{g} / \mathrm{mL}$ ) at $50 \%$ of individual maximal response multiplied by 100 .

tibodies to wasp venom in relation to the total IgE tended to be higher in high-sensitivity subgroups (median [range]: $16.9 \%$ [5.9-34.6] vs. $5.7 \%$ [1-92.7]), but these differences did not reach statistical significance $(\mathrm{p}=0.05$; Fig. 7c). There was no correlation between IgE humoral parameters and the basophil response to wasp venom. In the high-sensitivity subgroup, 3 wasp-allergic subjects experienced the most severe Mueller grade IV reaction, and 2 experienced grade III, and 2 grade II reactions. By contrast, in the low-sensitivity subgroup, only 1 subject experienced the Mueller grade IV reaction, 3 grade III, 1 grade II and 3 grade I reactions. Despite the tendency to a more severe reaction in the high-sensitivity subgroup, the differences did not reach statistical significance $(p \geq 0.07)$.

In wasp venom-allergic donors whose basophils were used for passive IgE sensitisation, we compared IgE and FceRI cell surface expression and the severity of sting reactions. In the group of 7 donors with similar IgE levels (range: $97-166 \times 10^{3}$ ) and FceRI numbers (range: 87 $141 \times 10^{3}$ molecules per basophil), 2 subjects experienced Mueller grade IV reaction, 2 grade III, 2 grade II, and 1 grade I reactions. A donor with a lower IgE (range: $23 \times$ $10^{3}$ ) and FceRI (range: $26 \times 10^{3}$ ) molecules per basophil experienced a Mueller grade IV reaction. There was no correlation between IgE or FceRI cell surface expression and basophil responses to wasp venom and specific $\operatorname{IgE}$ to wasp venom and $\mathrm{rVes} v 5$. The only significant, highly positive correlation was between IgE and FceRI cell surface expression ( $\mathrm{R}$ coefficient of $0.91, p=0.005$ ).

Distinct Factors Determine

Basophil-Allergen Sensitivity
In the high-sensitivity grass pollen-allergic subgroup, serum-specific IgE to timothy grass was higher than that in the low-sensitivity subgroup (median [range]: 25.6 kU/L [6.7-91.6] vs. $10 \mathrm{kU} / \mathrm{L}$ [1.3-50.1]; Fig. 7b, $p=0.04$ ), but these differences did not show statistical significance for $\mathrm{rPhl} \mathrm{p} \mathrm{1,} 5$ (median $30.4 \mathrm{kU} / \mathrm{L}$ [6.8-72.4] vs. $11.9 \mathrm{kU} / \mathrm{L}$ [0.4-60.6]; $p=0.08)$. No differences were found for the total $\operatorname{IgE}(267 \mathrm{kU} / \mathrm{L}$ [48-478] vs. $161 \mathrm{kU} / \mathrm{L}[105-1,168])$. However, the percentage of IgE antibodies to timothy differed in relation to the total IgE (median [range]: $14.6 \%$ [5-36] vs. $4.7 \%$ [1-17]; $p=0.02$ ). There was also a significant correlation between specific IgE antibodies or the grass-specific IgE fraction and the basophil response at 0.1 and $1 \mu \mathrm{g} / \mathrm{mL}$ of allergen or CD-sens ( $R$ coefficient $0.47-0.65 ; p \leq 0.04)$.

\section{Discussion}

IgE arms basophils, and mast cells can be activated within seconds of exposure to small amounts of allergens, yet the underlying factors that determine the sensitivity threshold for this activation are not completely understood. Using passive IgE sensitisation, we explored the relative role of allergen-specific IgE in this cellular response, as the IgE antibodies from the patient's sera were transferred to the stripped donor basophils, and the donor basophil response was compared with the initial responses of the patient or donor basophils. This study design demonstrated that in anaphylactic Hymenoptera venom-allergic patients, the intrinsic cellular sensitivity appears to be a key factor contributing to the sensitivity of the basophil response. By contrast, in rhinitis patients, the magnitude of the basophil sensitivity was the consequence of the allergen-specific IgE ratio on the cell surface.

The major observation of this study was that when we tested healthy donor basophils sensitised with the IgE antibodies of different wasp venom-allergic subjects, the IgE antibodies of subjects with an initial high or low basophil sensitivity induced a highly comparable responsiveness. Furthermore, the donor basophils of anaphylactic wasp venom-allergic subjects demonstrated after passive sensitisation with unrelated house dust mite or wasp venom IgE antibodies, the same cellular sensitivity or CD-sens as was initially recognized. This unexpected behaviour suggests that the basophil intrinsic sensitivity contributed considerably to Hymenoptera venom allergen cellular sensitivity and that the antigen-specific IgE or FceRI receptor density on the cell surface seems not to be the ma- 


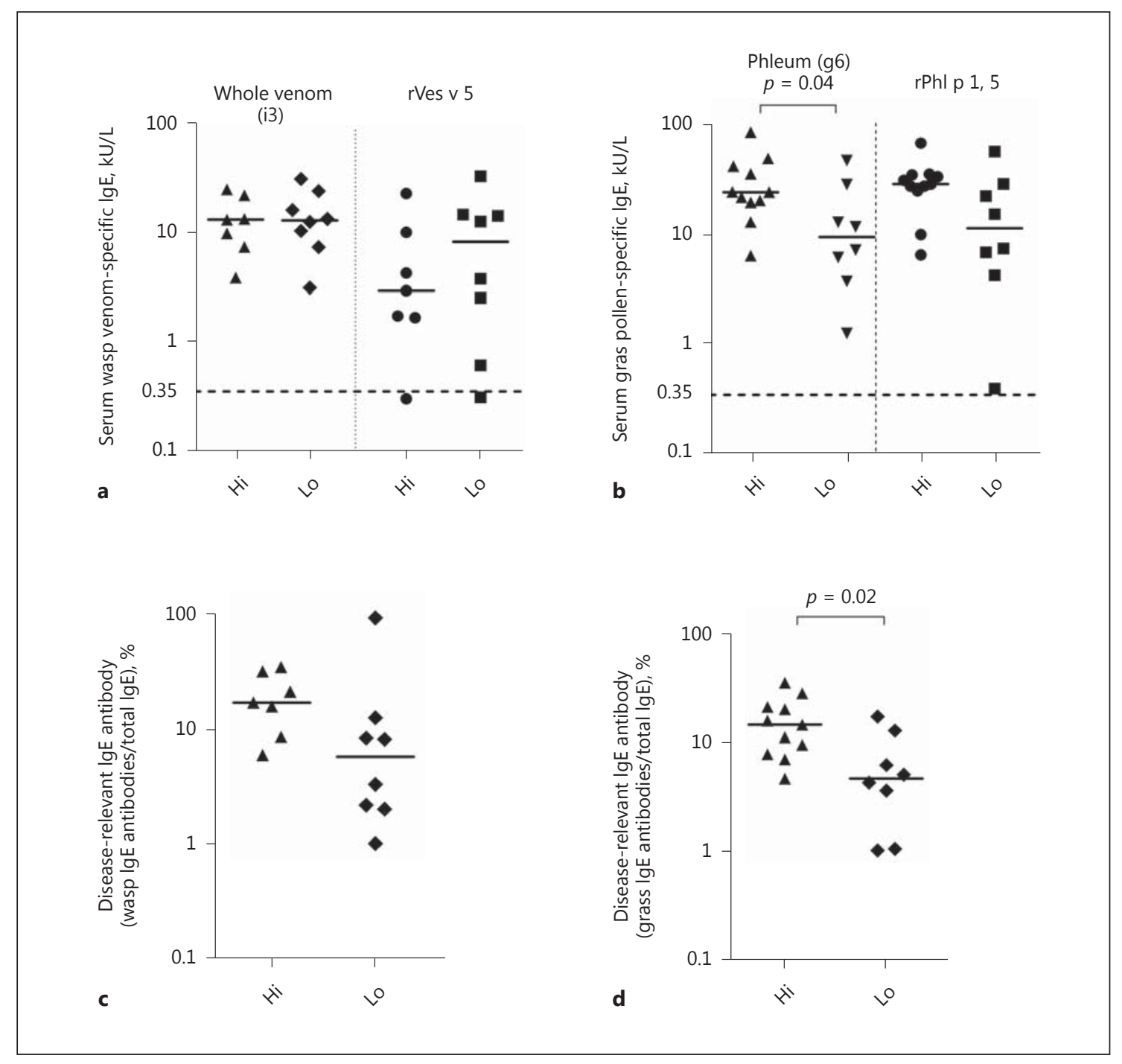

Fig. 7. Comparison between specific IgE to wasp venom and rVes v 5 (a) or Phleum and rPhl p 1, 5 (b) and the specific IgE to wasp venom (c) or Phleum /total IgE ratio (d) in the sera of 7 wasp-allergic (WA) subjects who showed initially high basophil allergen sensitivity and 9 WA subjects with initially low basophil allergen sensitivity $(\mathbf{a}, \mathbf{c})$, or in the sera of 11 grass-allergic (GA) subjects who showed initially high basophil allergen sensitivity and 8 GA subjects with initially low basophil-allergen sensitivity (b, d). Hi, high sensitivity; Lo, low sensitivity. The horizontal line indicates the median.

jor factor contributing to the size of the basophil response in this disease. IgE antibodies might be more important in patients with low basophil allergen sensitivity, and this trend was also observed in our report. Those findings provide novel insights into the nature of cellular responses in anaphylactic Hymenoptera allergy and fit well into the current knowledge about the lack of a correlation between IgE antibody levels and allergen sensitivity in this disease. Namely, it has been repeatedly demonstrated that differences in specific IgE levels or specific/total IgE ratios are not linked to the presence, absence, or severity of the clinical response to Hymenoptera stinging [10-13]. Moreover, up to $30 \%$ of the population are IgE sensitised to Hymenoptera venom, but fewer than $4 \%$ react in an anaphylactic way, and the majority of those sensitised individuals remain asymptomatic [28]. A recent report also demonstrated that an increase in specific IgE levels as induced by a sting challenge of asymptomatically sensitised 
subjects does not increase the risk of developing anaphylactic reactions to future stings [29]. In addition, previous reports have clearly described patients with a severe Hymenoptera venom allergy but with a negative or very low level of serum venom-specific IgE $[11,14]$. Furthermore, we demonstrated that up to $60 \%$ of patients with severe and/or repeated sting reactions but negative venom-specific IgE and skin test had a positive allergen-specific basophil response [14].

In patients with grass pollen-allergic rhinitis, we demonstrated that the extent of specific IgE sensitisation, especially the specific/total IgE ratio, significantly correlated with both the initial patient's basophil allergen sensitivity and the sensitivity of healthy donor basophils after passive IgE sensitisation. These results suggest that in grass pollen allergy, the basophil sensitivity is preferably determined by the degree of IgE sensitisation, which reflects the cell density of the antigen-specific IgE. Another factor that might also influence those correlations is a different affinity of specific IgE [30]; however, this factor was not tested in the current study. A significant positive correlation between basophil sensitivity and the extent of grass pollen-specific IgE sensitisation was also evident in our previous report [19]. Similar results were also observed after in vivo passive sensitisation of patients receiving a plasma transfusion containing IgE antibodies to a timothy grass pollen allergen [2]. Namely, in this report, the transfused IgE antibodies had sensitised basophils to an allergen sensitivity level that was similar to those of allergic plasma donor patients.

The relationship between different factors contributing to the basophil allergen response was recently explored using omalizumab to manipulate IgE levels. In asthma and/or rhinitis patients allergic to cat, grass pollen or house dust mites, downregulation of the IgE factor by anti-IgE treatment markedly reduced both basophil responses and clinical signs $[6,7,31]$. During omalizumab treatment, symptoms reduce when the basophil but not the mast cell response was reduced [7]. The omalizumab experimental design was also extended to subjects with anaphylactic peanut allergy. Unexpectedly, in peanut allergy, despite the decreased antigen-specific IgE densities during anti-IgE treatment, the majority of the subjects' basophil responses to peanut allergen did not decrease [8, 9]. The key determinant that opposes the effect of the downregulation of the IgE factor and causes a failure to suppress the basophil response, which was also inversely correlated with improvement in the patient's ability to tolerate peanut ingestion, was the increased intrinsic sensitivity of basophils to IgE-mediated stimulation [9].
These observations appear to be similar to our observations and speak in favour of differences in the characteristics of cellular responses in different allergic diseases. Namely, both the omalizumab $[6,7,31]$ and the current passive sensitisation study suggest that in subjects with cat, mite or pollen allergy, the differences or changes in the cell surface density of specific IgE shift the magnitude of the basophil response, which is a logical link because the IgE density determines how readily an antigen can reach levels for optimal stimulation. However, in patients with anaphylactic Hymenoptera or peanut allergy, this pattern was considerably interrupted by the influence of intrinsic cellular sensitivity. The basis and underlying mechanisms for these differences are not yet known, although some authors indicate that Syk expression and some other early elements might efficiently change and/ or regulate intrinsic basophil sensitivity $[5,32]$. Unfortunately, we did neither titrate the response to anti-FceRI mAbs, as described recently [23], nor evaluated if the basophil sensitivity to anti-FceRI stimulation is related with allergen basophil sensitivity. Anti-FceRI and fMLP were only used as positive control stimuli, as we described previously $[3,14,19,26,27]$, to confirm if the study patients and controls were basophil responders. Another limitation of this study was that healthy donor basophils and part of the wasp venom-allergic donor basophils were only tested with 2 wasp venom log concentrations. However, this was upgraded in house dust mite experiments, in which we used whole response curves with up to 6 wasp venom concentrations and $\mathrm{CD}$-sens calculation.

A recent report demonstrated that basophil allergen sensitivity is a potential marker of clinical airway allergen sensitivity in asthma patients [33]. Furthermore, during pollen or Hymenoptera venom immunotherapy, basophil allergen sensitivity is significantly decreased $[26,27$, 34-36]. In pollen immunotherapy, the obvious reason for these decreases is the induction of allergen-specific IgG antibodies with inhibitory activity against IgE-facilitated binding, and these antibodies showed sustained disease and cell-modifying effects that persisted for years after the discontinuation of immunotherapy [35-37]. By contrast, in the Hymenoptera venom immunotherapy model, blocking IgG antibodies seems to be of low or no importance $[38,39]$, especially for long-term cellular changes, making it likely that other intrinsic mechanisms are responsible for the suppression of basophil sensitivity $[26,27]$. These differences further suggest the importance of IgE allergen binding for the basophil response in pollen allergy and the importance of cellular intrinsic factors for the basophil response in Hymenoptera venom allergy. 
This study was not designed to compare the severity of clinical symptoms with the patient's basophil allergen sensitivity. Nevertheless, the most severe sting reactors were apparently more often in the high-sensitivity subgroup ( 3 of 4 with Mueller grade IV reactions, but only 2 of 5 with Mueller grade III reactions), whereas mild systemic reactors were only found in the low-sensitivity subgroup (all 3 with Mueller grade I reaction). In the anaphylactic Hymenoptera venom hypersensitivity model, no study has comprehensively compared the relationship between the severity of the reaction and basophil sensitivity. Previously, Erdmann et al. [40] demonstrated that basophil reactivity did not correlate with the protective effect of venom immunotherapy. In addition, several studies have reported that venom immunotherapy primarily modified basophil sensitivity [26, 27]. However, in the anaphylactic peanut model, patients who developed symptoms in an oral food challenge to peanut had higher basophil sensitivity than patients who tolerated provocation $[41,42]$, and recently it was even suggested that distinct parameters of the basophil CD63 response reflect the severity and threshold of allergic reactions to peanut $[43,44]$.
In grass pollen-allergic patients, we did not perform nasal allergen challenge experiments or a diary evaluation of the symptom and medication scores during the grass pollination season permitting a comparison of the basophil allergen sensitivity with the severity of the rhinitis symptoms. However, previous studies suggested that basophil allergy sensitivity correlates with the nasal provocation titre and the appearance of clinical symptoms in allergic rhinitis $[6,19]$ and with the allergen-specific bronchial provocation threshold in allergic asthma [33]. Furthermore, patients with allergic rhinitis confirmed by nasal provocation with no detectable specific IgE or skin testing but a positive basophil response are a notable example [45].

In summary, these studies demonstrate that basophil allergen sensitivity depends on different contribution factors in different allergic diseases. In anaphylactic Hymenoptera allergy, basophil allergen sensitivity was largely determined by cellular sensitivity to IgE-mediated stimulation. In allegic rhinitis triggered by pollens of seasonal plants, the major factor that determined the basophil allergen sensitivity was the variability in the activity of allergen-specific IgE.

\section{References}

1 MacGlashan DW Jr: Releasability of human basophils: cellular sensitivity and maximal histamine release are independent variables. J Allergy Clin Immunol 1993;91:605-615.

2 Johansson SG, Nopp A, van Hage M, Olofsson N, Lundahl J, Wehlin L, Söderström L, Stiller V, Oman H: Passive IgE-sensitization by blood transfusion. Allergy 2005;60:11921199.

3 Košnik M, Šilar M, Bajrovič N, Mušič E, Korošec P: High sensitivity of basophils predicts side-effects in venom immunotherapy. Allergy 2005;60:1401-1406.

4 Saini SS, Richardson JJ, Wofsy C, LavensPhillips S, Bochner BS, Macglashan DW Jr: Expression and modulation of FceRIa and FceRI $\beta$ in human blood basophils. J Allergy Clin Immunol 2001;107:832-841.

5 MacGlashan DW Jr: Relationship between Syk and SHIP expression and secretion from human basophils in the general population. J Allergy Clin Immunol 2007;119:626-633.

6 Nopp A, Johansson SG, Ankerst J, Bylin G, Cardell LO, Grönneberg R, Irander $\mathrm{K}$, Palmquist M, Oman $\mathrm{H}$ : Basophil allergen threshold sensitivity: a useful approach to anti-IgE treatment efficacy evaluation. Allergy 2006;61:298-302.

7 Eckman JA, Sterba PM, Kelly D, Alexander V, Liu MC, Bochner BS, Macglashan DW Jr, Saini SS: Effects of omalizumab on basophil and mast cell responses using an intranasal cat allergen challenge. J Allergy Clin Immunol 2010;125:889-895.

8 Savage JH, Courneya JP, Sterba PM, Macglashan DW, Saini SS, Wood RA: Kinetics of mast cell, basophil, and oral food challenge responses in omalizumab-treated adults with peanut allergy. J Allergy Clin Immunol 2012; 130:1123-1129.

9 Macglashan DW Jr, Savage JH, Wood RA, Saini SS: Suppression of the basophil response to allergen during treatment with omalizumab is dependent on 2 competing factors. J Allergy Clin Immunol 2012;130:1130-1135.

10 Day J, Buckeridge D, Welsh A: Risk assessment in determining systemic reactivity to honeybee stings in sting-threatened individuals. J Allergy Clin Immunol 1994;93:691-705.

11 Golden DB, Kagey-Sobotka A, Norman PS, Hamilton RG, Lichtenstein LM: Insect sting allergy with negative venom skin test responses. J Allergy Clin Immunol 2001;107:897901.

12 Korosec P, Valenta R, Mittermann I, Celesnik N, Erzen R, Zidarn M, Kosnik M: Low sensitivity of commercially available rApi $\mathrm{m} 1$ for diagnosis of honeybee venom allergy. J Allergy Clin Immunol 2011;128:671-673.

13 Korošec P, Valenta R, Mittermann I, Celesnik N, Silar M, Zidarn M, Košnik M: High sensitivity of CAP-FEIA rVes v 5 and rVes v 1 for diagnosis of Vespula venom allergy. J Allergy Clin Immunol 2012;129:1406-1408.

14 Korosec P, Erzen R, Silar M, Bajrovic N, Kopac P, Kosnik M: Basophil responsiveness in patients with insect sting allergies and negative venom-specific immunoglobulin $\mathrm{E}$ and skin prick test results. Clin Exp Allergy 2009; 39:1730-1737.

15 Hoffman DR: Fatal reactions to hymenoptera stings. Allergy Asthma Proc 2003;24:123127.

16 Pastorello EA, Incorvaia C, Ortolani C, Bonini S, Canonica GW, Romagnani S, Tursi A, Zanussi C: Studies on the relationship between the level of specific IgE antibodies and the clinical expression of allergy: I. Definition of levels distinguishing patients with symptomatic from patients with asymptomatic allergy to common aeroallergens. J Allergy Clin Immunol 1995;96:580-587.

17 Twardosz-Kropfmüller A, Singh MB, Niederberger V, Horak F, Kraft D, Spitzauer S, Valenta R, Swoboda I: Association of allergic patients' phenotypes with IgE reactivity to recombinant pollen marker allergens. Allergy 2010;65:296-303.

18 Movérare R, Petays T, Vartiainen E, Haahtela $\mathrm{T}$ : IgE reactivity pattern to timothy and birch pollen allergens in Finnish and Russian Karelia. Int Arch Allergy Immunol 2005;136:3338. 
19 Zidarn M, Košnik M, Silar M, Grahek A, Korošec $\mathrm{P}$ : Rhinitis symptoms caused by grass pollen are associated with elevated basophile allergen sensitivity and a larger grass-specific immunoglobulin E fraction. Clin Exp Allergy 2012;42:49-57.

20 Ebo DG, Hagendorens MM, Bridts CH, De Clerck LS, Stevens WJ: Sensitization to crossreactive carbohydrate determinants and the ubiquitous protein profilin: mimickers of allergy. Clin Exp Allergy 2004;34:137-144.

21 Pruzansky JJ, Grammer LC, Patterson R, Roberts M: Dissociation of IgE from receptors on human basophils. I. Enhanced passive sensitization for histamine release. J Immunol 1983;131:1949-1953.

22 Wallowitz ML, Chen RJ, Tzen JT, Teuber SS: Ses i 6, the sesame 11S globulin, can activate basophils and shows cross-reactivity with walnut in vitro. Clin Exp Allergy 2007;37: 929-938.

23 Čelesnik Smodiš N, Šilar M, Eržen R, Rijavec $M$, Košnik $M$, Korošec $P$ : Down-regulation of FceRI-mediated CD63 basophil response during short-term VIT determined venomnonspecific desensitization. PLoS One 2014; 9:e94762.

24 Nopp A, Johansson SG, Ankerst J, Palmqvist $\mathrm{M}$, Oman $\mathrm{H}$ : CD-sens and clinical changes during withdrawal of Xolair after 6 years of treatment. Allergy 2007;62:1175-1181.

25 Johansson SG, Nopp A, Oman H, Ankerst J, Cardell LO, Grönneberg R, Matsols H, Rudblad S, Strand V, Stålenheim G: The size of the disease relevant IgE antibody fraction in relation to 'total-IgE' predicts the efficacy of anti-IgE (Xolair) treatment. Allergy 2009;64: 1472-1477.

26 Eržen R, Košnik M, Silar M, Korošec P: Basophil response and the induction of a tolerance in venom immunotherapy: a long-term sting challenge study. Allergy 2012;67:822-830.

27 Žitnik SE, Vesel T, Avčin T, Šilar M, Košnik $\mathrm{M}$, Korošec P: Monitoring honeybee venom immunotherapy in children with the basophil activation test. Pediatr Allergy Immunol 2012;23:166-172.
28 Golden DB, Marsh DG, Kagey-Sobotka A, Freidhoff L, Szklo M, Valentine MD, Lichtenstein LM: Epidemiology of insect venom sensitivity. JAMA 1989;262:240-244.

29 Sturm GJ, Kranzelbinder B, Schuster C, Sturm EM, Bokanovic D, Vollmann J, Crailsheim K, Hemmer W, Aberer W: Sensitization to Hymenoptera venoms is common, but systemic sting reactions are rare. J Allergy Clin Immunol 2014;133:1635-1643.

30 Christensen LH, Holm J, Lund G, Riise E, Lund K: Several distinct properties of the $\operatorname{IgE}$ repertoire determine effector cell degranulation in response to allergen challenge. J Allergy Clin Immunol 2008;122:298-304.

31 Nopp A, Johansson SG, Ankerst J, Palmqvist $\mathrm{M}$, Oman $\mathrm{H}$ : CD-sens and clinical changes during withdrawal of Xolair after 6 years of treatment. Allergy 2007;62:1175-1181.

32 Kepley CL: Antigen-induced reduction in mast cell and basophil functional responses due to reduced Syk protein levels. Int Arch Allergy Immunol 2005;138:29-39.

33 Dahlén B, Nopp A, Johansson SG, Eduards M, Skedinger M, Adédoyin J: Basophil allergen threshold sensitivity, CD-sens, is a measure of allergen sensitivity in asthma. Clin Exp Allergy 2011;41:1091-1097.

34 Konradsen JR, Nordlund B, Nilsson OB, van Hage M, Nopp A, Hedlin G, Grönlund H: High basophil allergen sensitivity (CD-sens) is associated with severe allergic asthma in children. Pediatr Allergy Immunol 2012;23: 376-384.

35 Nopp A, Cardell LO, Johansson SG, Oman H: CD-sens: a biological measure of immunological changes stimulated by ASIT. Allergy 2009;64:811-814

36 Lalek N, Kosnik M, Silar M, Korosec P: Immunoglobulin G-dependent changes in basophil allergen threshold sensitivity during birch pollen immunotherapy. Clin Exp Allergy 2010;40:1186-1193.

37 James LK, Shamji MH, Walker SM, Wilson DR, Wachholz PA, Francis JN, Jacobson MR, Kimber I, Till SJ, Durham SR: Long-term tolerance after allergen immunotherapy is accompanied by selective persistence of blocking antibodies. J Allergy Clin Immunol 2011; 127:509-516.
38 Varga EM, Francis JN, Zach MS, Klunker S, Aberer W, Durham SR: Time course of serum inhibitory activity for facilitated allergen-IgE binding during bee venom immunotherapy in children. Clin Exp Allergy 2009;39:13531357.

39 Möbs C, Müller J, Rudzio A, Pickert J, Blank S, Jakob T, Spillner E, Pfützner W: Decline of Ves $\mathrm{v}$ 5-specific blocking capacity in wasp venom-allergic patients after stopping allergen immunotherapy. Allergy 2015;70:715-9.

40 Erdmann SM, Sachs B, Kwiecien R, MollSlodowy S, Sauer I, Merk HF: The basophil activation test in wasp venom allergy: sensitivity, specificity and monitoring specific immunotherapy. Allergy 2004;59:1102-1109.

41 Glaumann S, Nopp A, Johansson SG, Rudengren M, Borres MP, Nilsson C: Basophil allergen threshold sensitivity, CD-sens, IgEsensitization and DBPCFC in peanut-sensitized children. Allergy 2012;67:242-247.

42 Santos AF, Douiri A, Bécares N, Wu SY, Stephens A, Radulovic S, Chan SM, Fox AT, Du Toit G, Turcanu V, Lack G: Basophil activation test discriminates between allergy and tolerance in peanut-sensitized children. J Allergy Clin Immunol 2014;134:645-652.

43 Homšak M, Silar M, Berce V, Tomazin $M$, Skerbinjek-Kavalar M, Celesnik N, Košnik M, Korošec P: The relevance of basophil allergen sensitivity testing to distinguish between severe and mild peanut-allergic children. Int Arch Allergy Immunol 2013;162:310-317.

44 Santos AF, Du Toit G, Douiri A, Radulovic S Stephens A, Turcanu V, Lack G: Distinct parameters of the basophil activation test reflect the severity and threshold of allergic reactions to peanut. J Allergy Clin Immunol 2015;135: 179-186.

45 Gómez E, Campo P, Rondón C, Barrionuevo E, Blanca-López N, Torres MJ, et al: Role of the basophil activation test in the diagnosis of local allergic rhinitis. J Allergy Clin Immunol 2013;132:975-976.e5.
Distinct Factors Determine

Basophil-Allergen Sensitivity
Int Arch Allergy Immunol 2016;171:89-101 